\title{
Espacio geográfico y fenómeno técnico: cuestiones de método
}

María Laura Silveira

CONICET / Instituto de Geografía, Facultad de Filosofía y Letras, Universidad de Buenos Aires, Argentina.

Recibido: 22 de diciembre de 2017. Aceptado: 30 de agosto de 2018.

\begin{abstract}
Resumen
La categoría de espacio geográfico puede ser definida y operacionalizada si consideramos a la técnica como un elemento constitutivo del espacio. En primer lugar, interesa discutir no solamente las técnicas particulares sino fundamentalmente el fenómeno técnico, que incluye los objetos técnicos, sus modos de uso y la política subyacente en sus combinaciones y localizaciones. Así la técnica puede ser vista como un medio, en el cual los lugares revelan contenidos técnicos diversos. En otras palabras, es un esfuerzo de ir más allá de las técnicas particulares con el propósito de discutir una fenomenología de la técnica, que sea vista como base para una explicación geográfica. En segundo lugar, las técnicas permiten empirizar al tiempo, alejando el riesgo de ver el tiempo separado del espacio. Así, la periodización como recurso de método posibilitaría comprender los usos del espacio en cada momento de la historia y, en consecuencia, alcanzar una perspectiva diacrónica que no sea abstracta. En tercer lugar, analizaremos las técnicas contemporáneas, es decir, la naturaleza sistémica de las técnicas de la globalización, allí incluidas las técnicas de la información, sus escalas, sus condiciones de rigidez y flexibilidad, su velocidad de transformación y su indisolubilidad con la acción, la norma y la ideología.
\end{abstract}

PALABRAS CLAVE: FENÓMENO TÉCNICO. ESPACIO GEOGRÁFICO. TIEMPO. PERÍODO. GLOBALIZACIÓN.

\section{Geographical space and technical phenomenon: questions of method}

\begin{abstract}
The category of geographical space could be defined and operationalized considering the technique as a constitutive element of space. Firstly, we will not only consider the particular techniques, but essentially the technical phenomenon that includes objects, forms of usage, underlying politics and its particular locations. This leads us to understand the technique as an environment, in which the places reveal diverse technical
\end{abstract}


contents. In other words, we propose an effort to go beyond the particular techniques with the purpose to discuss a phenomenology of the technique as a basis for a geographical explanation. Secondly, the techniques allow us to understand time as an empirical fact, avoiding seeing the time and the space as separated dimensions. In this regard, periodization is an essential element of the method that allows to apprehend the usages of space in every moment of history, and as a consequence to reach a non-abstract diachronic perspective. Thirdly, we will analyze the contemporary techniques, i.e., the systemic nature of techniques in globalization, including information techniques, scale, speed and conditions of rigidity and flexibility of technique and their inseparability of action, norm and ideology.

KEYWORDS: TECHNICAL PHENOMENON. GEOGRAPHICAL SPACE. TIME. PERIOD. GLOBALIZATION.

PALAVRAS-CHAVE: FENÔMENO TÉCNICO. ESPAÇO GEOGRÁFICO. TEMPO. PERÍODO. GLOBALIZAÇÃO.

\section{Introducción}

Frente a la complejidad del mundo contemporáneo se vuelve indispensable reflexionar sobre las categorías de la disciplina con el propósito de actualizarlas y, de ese modo, alcanzar su operacionalización en la investigación. Aquí nos detenemos en la discusión de las categorías espacio geográfico y técnica siendo ésta vista, al mismo tiempo, como fenómeno y como medio. Nos interesa discutir fundamentalmente el fenómeno técnico, que incluye los objetos técnicos, sus modos de uso y la política subyacente en sus combinaciones y localizaciones. Así la técnica puede ser vista como un medio, en el cual los lugares revelan contenidos técnicos diversos. En otras palabras, es un esfuerzo de ir más allá de las técnicas particulares con el propósito de discutir una fenomenología de la técnica, que sea vista como base para una explicación geográfica.

En segundo lugar, pensamos que en su doble condición de materialidad e inmaterialidad, la técnica permite empirizar al tiempo y, de ese modo, reconocer los eventos, alejando el riesgo de ver el tiempo separado del espacio. Así, la periodización como recurso de método posibilitaría comprender los usos del espacio en cada momento de la historia y, en consecuencia, alcanzar una perspectiva diacrónica que no sea abstracta.

Finalmente, discutimos algunos trazos de las técnicas que constituyen la base material y organizacional de la globalización, es decir, la naturaleza sistémica de las técnicas contemporáneas, allí incluidas las técnicas de la información, sus escalas, sus condiciones de rigidez y flexibilidad, su velocidad de transformación y su indisolubilidad con la acción, la norma y la ideología.

\section{Geografía, espacio y fenómeno técnico}

La historia de la Geografía es rica en estudios dedicados a la descripción de instrumentos técnicos y herramientas en diferentes porciones de la superficie terrestre, permeados por los debates sobre medio geográfico y género de vida y, en definitiva, por la relación hombre-medio tan frecuente en las teorías clásicas (Silveira, 2012). Más tarde, y especialmente en vertientes anglosajonas, el énfasis fue dado a la relación entre una técnica determinada y el espacio, originando un conjunto de geografías particulares vinculadas 
Espacio geográfico y fenómeno técnico: cuestiones... MARÍA LAURA SILVEIRA

al transporte, a las comunicaciones, a las industrias y a otras variables significativas. Ambos esfuerzos parecen poner de manifiesto el status que la técnica ha alcanzado en la tradición de la disciplina pero, fundamentalmente, muestran el valor del análisis de las diversas manifestaciones de la técnica en el espacio geográfico.

Entretanto tal vez no es pertinente ver, en tales ejercicios, una intención por valorizar a la técnica como fenómeno constitutivo del espacio geográfico. La identificación de aquello que Sartre (1968: 17) denomina "condición fenoménica”, es decir, que algo existe sólo en cuanto se revela, no implica una reflexión sobre el fenómeno en sí mismo. No se trata, sin embargo, de imaginar que "el fenómeno sea una apariencia que remite a un ser distinto" o, en otras palabras, que "el ser se encuentre escondido tras los fenómenos" (Sartre, 1968: 16).

En esa línea de reflexión podríamos decir que, vistas como particulares, las técnicas serían tal vez aprehendidas como condiciones fenoménicas, como instrumentos u operaciones técnicas y no necesariamente como un fenómeno técnico. Según Ellul (1968: 18) en las formas diferentes de trabajar es posible encontrar "algunos puntos comunes, ciertas tendencias, ciertos principios, idénticos en todas partes”, pero no es aconsejable denominarlos "Técnica con T mayúscula". De lo que se trata, continúa el filósofo, es de buscar el método más eficaz, el reino del pensamiento y la razón, el "fenómeno técnico, hoy universal" (Ellul, 1968: 18). En su obra originalmente publicada en 1996, Santos ya escribía que una técnica particular, entendida "como un medio de conseguir éste o aquel resultado específico" conduce a nociones particulares del espacio, es decir, espacio agrícola, espacio industrial y espacio económico, por citar sólo algunas (2000: 33). Y agrega "sólo el fenómeno técnico en su total comprensión permite alcanzar la noción de espacio geográfico" (2000: 33). Se trataría, entonces, de ir más allá de las técnicas particulares porque, como explica Simmel:

Indudablemente un hondo problema reside en que fenómenos de existencia individual e independiente, apartándose los unos de los otros conforme determinaciones espaciales temporales objetivas a discreción, coinciden sin embargo en una determinación cualquiera, que deben ellos tener en común, que su total incomparabilidad en todas partes no puede impedir su identidad en un punto (2006: 70).

Buscando reconocer esa identidad a que se refiere Simmel, en la multiplicidad de técnicas particulares a lo largo de la historia de la humanidad, y particularmente en el presente, diríamos que la concepción de la técnica como conjunto interdependiente de objetos y formas de uso parece un camino posible. Más allá de su grado de complejidad técnica, todas las épocas tienen en común la indisolubilidad entre los instrumentos y la forma de manipularlos. Esa es la propuesta de Santos (2000), quien entiende a la técnica como un proceso originariamente externo al espacio -y por lo tanto una categoría externa a la geografía - que permite dinamizar categorías analíticas internas de la disciplina como paisaje, configuración territorial, división territorial del trabajo y espacio producido, entre otras. Así, el autor destaca la centralidad de la técnica porque, cuando ésta es entendida como un conjunto de objetos y usos en intrínseca relación con la política, es capaz de reunir categorías internas y externas de la disciplina. De ese modo, para Santos (2000) la técnica revela la producción histórica de la realidad, inspira un método unitario y no dualista, y es vista como garantía de la conquista del futuro. 
Espacio geográfico y fenómeno técnico: cuestiones... MARÍA LAURA SILVEIRA

En otras palabras, entendida como fenómeno, la técnica es forma y acción o evento. Ya Heidegger (1958) se refería a la técnica como una categoría inclusiva conformada por los instrumentos y máquinas creados, pero también por las necesidades y fines que animan a esa producción. Como forma, la técnica es un contenido material, un conjunto de objetos constituido por una pluralidad de instrumentos yuxtapuestos con funciones y valores diversos, subordinados y dependientes, que podríamos asociar a la noción de tecnología. ${ }^{1}$ Es un elemento constitutivo del espacio geográfico que lo diferencia del mero espacio social. En tanto acción o evento, la técnica es un conjunto de usos, procedimientos y normas que dan contenido previsible a buena parte de esas acciones. Este otro elemento constitutivo del espacio geográfico permite afirmar que es más que el simple espacio material o físico. En esa relación indisoluble, objetos técnicos y acciones tecnificadas posibilitan diversos tiempos de producción y circulación -material y simbólica- y, de esa manera, permiten reconocer actores y temporalidades distintas. Akrich (1987: 49) también asevera que "los objetos técnicos tienen un contenido político en el sentido que constituyen elementos activos de organización de relaciones de los hombres entre sí y con el medio", al mismo tiempo que "devienen mediaciones obligadas en todas las relaciones que establecemos con lo real". Para esa autora, el objeto técnico se define como la relación entre un dispositivo material y los usos cumplimentados por ese dispositivo (Akrich, 1987).

En la misma sintonía, Gras (1993) ha sido crítico en relación a cierta historia simplista de la tecnología, que aísla la evolución de los objetos técnicos sin aprehenderlos dentro del sistema de interrelaciones que éstos crean. Para el autor, desde su nacimiento, son objetos sociales y la evolución del contexto forma parte de la evolución del ser técnico (Gras, 1993). Aun concordando con las reflexiones que alertan para no olvidar los contextos en la historia de las técnicas, no podemos dejar de señalar una oposición recurrente: lo técnico y lo social, como si fuesen dos dimensiones de una realidad que entendemos como unitaria desde su mismo origen.

En ese sentido, la propuesta de ver a la técnica como fenómeno no se circunscribe a inventariar las técnicas en su condición fenoménica o como algo independiente del medio que las acoge, sino que supone la comprensión del proceso que resulta del encuentro, en el lugar, de las técnicas preexistentes y de las nuevas que llegan desigual y selectivamente. Ese conjunto heterogéneo y cambiante constituye un medio para las acciones que, a su vez, lo transformarán en otra cosa. Comprender la técnica como un medio supone reducir fenomenológicamente a los objetos técnicos, entendiendo que se trata de un tejido de cosas y nexos, siendo estos últimos menos visibles pero igualmente explicativos. Como afirma Santos al discutir las relaciones entre técnica y espacio:

Debemos partir del hecho de que esos diferentes sistemas técnicos forman una situación y son una existencia en un lugar dado, para tratar de entender, a partir de ese sustrato, cómo se realizan las acciones humanas. La forma en que se combinan sistemas técnicos de diferentes edades va a tener una

1 La sinonimia o la diferencia entre las nociones de técnica y tecnología conducen a un largo debate que no podemos desarrollar aquí. Preferimos, entretanto, entender a la técnica como el conjunto de los objetos y de sus formas de uso. La tecnología es vista aquí circunscrita a la materialidad. Un autor como Sigaut (1994) explica la difusión, a partir del mundo anglosajón, del vocablo tecnología como un modo de oponer los conjuntos técnicos modernos y científicos a las prácticas empíricas de los artesanos. Sin embargo, reconoce que en Alemania el término ya era utilizado en el siglo XVIII para referirse a una ciencia de las técnicas. 
consecuencia sobre las formas de vida posibles en aquel área. Desde el punto de vista específico de la técnica dominante, la cuestión es otra: verificar cómo los residuos del pasado son un obstáculo para la difusión de lo nuevo o cómo juntos encuentran la manera de permitir acciones simultáneas (2000: 38).

Esa reflexión lleva a una verdadera fenomenología de la técnica que es una base para la explicación geográfica, porque su objetivo no es aprehender técnicas particulares aisladas sino los sistemas que conforman y, en consecuencia, el nuevo medio que crean. Prades (1992: 18) afirma que la técnica "esposa" un medio, haciendo que todo se vuelva allí técnico, incluidos los modos de organización y las representaciones de la relación con el medio. En su opinión, no existiría tal realidad sin sistemas técnicos, es decir, conjuntos coherentes de objetos materiales e inmateriales y principios y soluciones técnicas. Perrin (1992) parece ir aún más lejos al señalar que las técnicas forman sistemas porque su concepción resulta de modelos organizacionales. Autores como Gille (1978) y Santos $(1985 ; 2000)$ han utilizado la noción de sistemas técnicos para destacar la interdependencia de las existencias técnicas. Por eso, podemos entender a los sistemas técnicos como conjuntos de instrumentos de trabajo que, después de ser resultado, se vuelven condición de la vida social y técnica. Así, los instrumentos de trabajo son espacio y crean espacio, a través de los sistemas de acciones que los rigen y les imponen una temporalidad. A ese proceso Ortega y Gasset lo considera la creación de una sobrenaturaleza, ya que la técnica es el conjunto de los actos técnicos, es decir, "lo contrario de la adaptación del sujeto al medio, puesto que es la adaptación del medio al sujeto" (1957: 17).

El geógrafo Pierre George también entendía la técnica más allá de la mera materialidad al afirmar que:

La influencia de la técnica sobre el espacio se ejerce de dos maneras y a dos escalas diferentes: la ocupación del suelo por las infraestructuras de las técnicas modernas, fábricas, minas, canteras, espacios reservados a la circulación, y las transformaciones generalizadas impuestas por el uso de la máquina y por la implementación de nuevos métodos de producción y de existencia (1974: 13).

Buscando recuperar el sentido original del vocablo tekné, Perrin (1992) se refiere a los conjuntos de medios que van desde los conocimientos y el saber-hacer, a los instrumentos y máquinas, las organizaciones como las empresas, las instituciones que fijan normas y las representaciones simbólicas que confieren ciertos valores a las técnicas. Picon (1994) coincide en que objetos, dispositivos y procedimientos se acompañan de saberes, saber-hacer y representaciones en lo que podría denominarse pensamiento técnico en sentido amplio. Como ya explicara Darcy Ribeiro (1975: 22) "encontramos en todas las culturas un cuerpo mínimo de conocimientos objetivos y de modos estandarizados de hacer [...] la lógica de las cosas se impone a las culturas, desafiándolas a desarrollarse mediante la percepción de sus principios y la adaptación a ellos”.

La técnica es un medio porque constituye un contenido central, material y organizacional de la vida social contemporánea. Pero además porque, como escribe Santos (2000: 36) "es el espacio el que determina los objetos: el espacio visto como un conjunto de objetos organizados según una lógica y utilizados (accionados) según una lógica” o, en otros términos, "el espacio redefine los objetos técnicos, a pesar de sus vocaciones 
originales, al incluirlos en un conjunto coherente donde la contigüidad obliga a actuar en conjunto y solidariamente". Aquí la palabra es entregada al espacio y no a los objetos per se, exorcizando una visión mecanicista o tecnológica del espacio.

No se trata de un determinismo, sino de dar el necesario status epistemológico a la nueva textura del espacio geográfico ya que, como expresaba André Fel (1978: 1062), "el progreso técnico revoluciona la geografía". De ese modo, tal vez podríamos alejarnos de escisiones tradicionales y poco explicativas en la disciplina como físico y humano, natural y social, con el propósito de aproximarnos al estudio del espacio a través de la idea de técnica y por medio del procedimiento intuitivo que Simmel (2002: 102) define como "una particular disposición de la mirada, gracias a la cual se realiza la escisión entre la forma y el contenido" de una realidad crecientemente híbrida. Escisión que, entretanto, será únicamente un momento del proceso de análisis, para luego alcanzar la síntesis asegurada por la categoría y la ontología del espacio geográfico.

\section{Hacia una empirización posible del tiempo}

Parafraseando a Heidegger cuando discutía la cosa, podríamos preguntarnos ¿qué es la técnica? y responder como él, que la técnica revela la "íntima unión de tiempo y espacio" (1975: 22). El fenómeno técnico permite empirizar al tiempo y cualificar la materialidad sobre la cual se dan las acciones (Santos, 2000). Empirizamos el tiempo cuando analizamos las posibilidades concretas que, a cada momento de la historia, permitieron la realización de las acciones. ¿Qué instrumentos técnicos permitieron las grandes navegaciones? ¿Cuáles son las bases tecnológicas que permiten la circulación del dinero a escala planetaria? Cualificamos la materialidad sobre la que se dan las acciones al preguntarnos qué actores y de cuáles imperios pudieron disponer de tales instrumentos, o qué actores de la sociedad contemporánea pueden utilizar los dispositivos tecnológicos que permiten los flujos internacionales de dinero y cuánto deben pagar por esos procedimientos. En su discusión sobre la técnica y la ciencia, y lo indispensable de verlas como un proceso, Coriat (1976: 59) alertaba sobre la necesidad de examinar el "conjunto de las condiciones reales" y recordaba que "reales" para Marx significaba "antes de más nada materiales". Podríamos decir que las acciones de un agente son más eficaces y rápidas si se desarrollan sobre formas materiales modernas.

El reconocimiento de las posibilidades concretas demanda, sin embargo, un recurso de método, es decir, la escisión del tiempo histórico. Cuando buscamos dividir el tiempo -que es continuo- en partes, necesitamos encontrar un criterio que no mutile la indisolubilidad entre materialidad y acción, elementos constitutivos del fenómeno técnico y del espacio geográfico. Por su contenido de materialidad el espacio aparece como algo concreto, empírico, fácilmente divisible a partir de la instalación de los objetos. Sin embargo, esa fórmula conduciría, una vez más, a reducir el espacio a un mero escenario o, como ya alertaba Smith (1990), al espacio visto como el sitio, la fundación estable que la historia mueve. Al contrario, si reconocemos su contenido inmaterial como algo autónomo, el riesgo sería asumir una noción de tiempo meramente cronológica. Es el problema de la empirización del tiempo. Sin enfrentarlo, sucumbiríamos una vez más a la vieja fórmula: la historia trata del tiempo y la geografía del espacio. 
Sin embargo, entre fenómeno técnico y espacio geográfico existiría una relación biunívoca porque ambos se corresponden directamente, ambos resultan de la acumulación de tiempos o de la empirización del tiempo y uno no se explica sin el otro. El contenido técnico del espacio es un contenido de tiempo.

Por esa razón, la delimitación de un periodo permitiría ver más claramente cómo las posibilidades de ese momento producen un espacio - un territorio usado- y unos sistemas de acciones que se desarrollan sobre esas formas técnicas, normativas y culturales heredadas - un territorio siendo usado (Santos, 1994; Santos y Silveira, 2001; Silveira, 2014)-. En otras palabras, la historia es una totalidad de acontecimientos continuos, que es el tiempo realizándose en los lugares, cuya interpretación demanda una periodización. De allí que la pregunta de método fundamental sea ¿̇cómo escindir esa totalidad en movimiento en periodos o sistemas temporales? Pensamos que un camino posible es el fenómeno técnico.

Periodizar significa elegir elementos constitutivos de nuestro objeto, que no son inmutables sino variables en el devenir y que tendrán diferentes capacidades de regencia en la historia. Entretanto, esos elementos no varían aisladamente, sino conjuntamente con el proceso por el cual una totalidad se torna otra. Como reflexiona Bagú (1986), el sucederse de la realidad social supone un engendrarse y encadenarse de pasajes relacionales. Para el autor se trata de múltiples secuencias, cada una revelando cambios cualitativos, pero con elementos que no se pierden sino que ingresan a otra secuencia, a otro tipo de realidad relacional. Es también el pensamiento de Barraclough (1983: 15) al escribir: "todas las especies de cosas perduran de un periodo a otro". De allí que la delimitación de periodos sea una atribución del investigador quien, sin apartarse de los fundamentos de la historia, podrá reconocer los eventos más significativos que le permitan comprender su problema de investigación. Interesan más las épocas que los marcos que las separan.

En la aurora de la historia, como afirma Braudel (1973: 29), "el hombre fue el único instrumento, el único motor al servicio del hombre, por consiguiente el único artesano de la civilización material". Con el paso de los siglos, el fenómeno técnico fue volviéndose más complejo y algunos autores acuñaron expresiones que pudieran retratar los mundos sucesivos. Ellul (1968) se refería a la técnica como un nuevo medio natural, al tiempo que Simondon (1989) y Friedmann (1966) proponían hablar de un medio técnico, y Reclus (1985), de sucesión de medios. La vida social ya no podía ser pensada fuera del fenómeno técnico.

Es la relación entre tiempo, espacio y técnica lo que constituye el núcleo de la cuestión. Y Elias lo expresa en esta excerpta de su libro publicado originalmente en 1984:

Mientras los enclaves humanos aumenten más en extensión y relativa autonomía (gracias a los procesos de urbanización, comercialización y mecanización), tanto mayor será su dependencia de instrumentos de factura humana para medir y regular el tiempo, y menor su dependencia de medidas temporales inhumanas y naturales, como los movimientos de la Luna, el cambio de estaciones, de la pleamar y la bajamar. En las sociedades actuales de elevada urbanización e industrialización, la relación entre el cambio de las unidades del calendario y el cambio de las estaciones del año, sin perderse 
por completo, se hace indirecta y floja, y en algunos casos, como entre mes y movimiento lunar, el nexo más bien ha desaparecido. Cada vez más los hombres viven en un mundo de símbolos que ellos mismos han elaborado y, sin hacerse absoluta, la autonomía de sus enclaves se vuelve enorme (2010: 63).

$\mathrm{Al}$ aprehender ese proceso de realización de la sociedad en el espacio, de concreción de las acciones en la materialidad y de reconocimiento de las posibilidades concretas de la materialidad para ser utilizada, la dialéctica deja de ser abstracta. Un tiempo cronológico y abstracto y un espacio material, muchas veces reducido a la idea de distancia, no permitían empirizar ni uno ni otro en una existencia unitaria. Cuando el evento transforma al objeto se diluye en él y, a su vez, el objeto es la posibilidad material de la acción. Materialidad y acción son conceptos que se niegan entre sí para recrear un concepto, la técnica, capaz de dar cuenta de una realidad unitaria donde la técnica está impregnada de vida y ésta no se desarrolla sin la técnica. Estaríamos, así, más cerca de evitar el peligro que Gurvitch (1962: 5) señalaba como "momificación" de la dialéctica, al referirse a la situación que adviene de la incapacidad de aprehender totalidades reales en marcha con todas sus tensiones, oposiciones y conflictos. El espacio no es otra cosa que una totalidad en marcha.

\section{Técnicas contemporáneas: sistemas inteligentes, creatividad en la escasez}

En nuestros días nadie duda de la extrema complejidad del fenómeno técnico aunque, quizás, el rasgo más destacado sea su relación intrínseca con la ciencia y la información. Para Ladrière (1977) la ciencia no es más un método puro de conocimiento, sino un sistema de acción porque provoca transformaciones tecnológicas y políticas. Ese sistema de acción adquiere hoy tal extensión que podríamos afirmar, con Latour (2008), que la ciencia hace coincidir sus límites con los del resto de los intercambios sociales.

La base material de la globalización se caracteriza por la interdependencia de las técnicas y su marcada eficacia. Ya a mediados del siglo pasado, Simondon (1989: 34) afirmaba que "el objeto técnico concreto es el que no está más en lucha consigo mismo y en el cual ningún efecto secundario perjudica el funcionamiento del conjunto" y que tiende hacia un estado que hará del ser técnico un sistema enteramente coherente y unificado. Debray y Finkielkraut (1994: 242-243) coinciden al afirmar que "los objetos van hacia su perfección", y señalan: "el mundo de la técnica es el de la compatibilidad, de la estandarización, sin lo cual no hay difusión ni circulación [...] la normalización es el pasaje obligatorio que hace del planeta un todo interconectado e intraconectado". O, en palabras de Fel (1978: 1067): "es de la difusión de una tecnología universable que se trata”.

Causa y consecuencia de la interconexión y de la interdependencia aumentada de los objetos técnicos, los macro-sistemas técnicos, como Joerges (1988) identifica a las técnicas actuales, tienden a ultrapasar fronteras de todo tipo y son, como señala Gras (1993), la base para un gran número de otros sistemas que no funcionarían sin ese soporte. Al mismo tiempo, existen micro-sistemas técnicos, gracias a la miniaturización de los objetos, que revolucionaron los controles técnicos de la vida económica y 
Espacio geográfico y fenómeno técnico: cuestiones... MARÍA LAURA SILVEIRA

social. La denominada Internet of Things (IoT) parece la manifestación más reciente y perfeccionada de tales micro-sistemas, potenciando aún más la posibilidad de automatizar las tareas, pero también de vigilar ya que, como alerta Han (2014: 103) "nos observan cosas que nos rodean".

A pesar de su tendencia a la unicidad (Santos, 2000), el sistema técnico actual enfrenta el problema que Hughes (2008) denomina salientes reversas que, aunque no se trate de un dato enteramente nuevo, en nuestros días alcanzó una velocidad sin precedentes. Para el autor, "en la medida que los sistemas tecnológicos se expanden, desarrollan salientes reversas" que son "componentes del sistema comparativamente retrasados, es decir, que no evolucionaron con los otros" (Hughes, 2008: 134). Hoy, más que nunca antes, la tensión entre salientes reversas e invención marca el ritmo de la evolución de los sistemas técnicos pues, como Tarde escribió hace más de un siglo, "las invenciones, sean cuales fueran, son el verdadero capital que la economía política busca definir” (2011: 175).

A partir de un sistema técnico planetario recibido de una generación por otra -hecho absolutamente novedoso en la historia de la humanidad que Santos (2000) denomina tiempo empírico- las relaciones y los contextos se amplían. No existiría tal tiempo universal sin la extrema compatibilidad tecnológica a la que se refiere Prades (1992). Esa es la sustancia material de las acciones contemporáneas. Aquí las palabras que Arendt escribió a mediados del siglo pasado adquieren nuevo sentido: "dado que siempre actuamos en una red de relaciones, las consecuencias de cada acto son ilimitadas, toda acción provoca no sólo una reacción sino una reacción en cadena” (2005: 105). Entretanto, esa posibilidad de multiplicar las relaciones, que podríamos denominar ampliación de los contextos (Santos, 2000), no se realizaría sin una cierta retracción de las cuestiones logo-teóricas y ontológicas ante interrogaciones operacionales (Prades, 1992). Como afirmaron Debray y Finkielkraut (1994: 233), vivimos una era de "rechazo de la abstracción y de las mediaciones: lo que cuenta es lo concreto, la imagen". De ese modo, el uso de las nuevas tecnologías se vuelve posible "por una organización de los intercambios, una racionalización abstracta de las conductas y una logística pensada en un centro (o en una unidad funcional equivalente)" (Gras, 1993: 26). El imperio de los interrogantes operacionales lleva a que "toda problemática, todas las problemáticas deben entrar en un sistema de problemas y de respuestas que van a incrementar cada día el control de la organización sobre la vida planetaria" (Auzias, 1971: 107). Por estas y otras razones Torres Ribeiro alerta:

Los avances de la técnica indican una clara bifurcación: de un lado, se socializa la posibilidad de acción planificada, casi obligatoria para todos; por otro, se densifica la base técnica de la acción planificadora, tendiendo a tornarla un objeto exclusivo, disputado por players selectos (2012: 117).

El medio técnico-científico-informacional es, por lo tanto, el rostro geográfico de la globalización (Santos, 1985). Muchos autores se han referido más recientemente al papel de la tecnociencia en la sociedad contemporánea, incluyendo aquellas visiones que parecen no imaginar usos alternativos para los objetos que nos rodean. Preocupada con la "mercantilización de todo lo vivo", Braidotti (2015: 76) menciona los "cuatro jinetes del apocalipsis contemporáneo: nanotecnologías, biotecnologías, tecnologías de la información y ciencias cognitivas", los cuales revelan “una economía política más compleja que conecta cuerpos y máquinas de manera más íntima” (Braidotti, 2015: 108). 
La información y sus soportes se vuelven entonces mercaderías valiosas. Explicando la implementación de la Internet 2.0 en el inicio del siglo XXI, Van Dijck señala el surgimiento de las plataformas interactivas que, proclamando la idea de participación, utilizan "sistemas automatizados que inevitablemente diseñan y manipulan las conexiones" (2016: 29). De esa manera, "siguen el rastro de sus deseos y reducen a algoritmos las relaciones entre personas, cosas e ideas" (Van Dijck, 2016: 29). Y agrega la autora "hacer social la red en realidad significa hacer técnica la socialidad", alcanzando una "socialidad tecnológicamente codificada" (Van Dijck, 2016: 30). La firma IBM creó recientemente la unidad IBM Cognitive Business Solutions, que utiliza tecnología cognitiva para prever tendencias de consumo a partir del Watson, su sistema de inteligencia artificial que puede interactuar con el lenguaje natural. Se trata de un nuevo modelo de computación que incluye analítica avanzada, procesamiento del lenguaje natural y aprendizaje de las máquinas.

Como explican Mattelart y Vitalis, la automatización de la recolección de informaciones y la co-producción de datos por parte de los consumidores, posibles gracias a la digitalización y al uso masivo de las redes, permiten que un puñado de monopolios privados ofrezcan "servicios que se monetizan en unidades de vida privada" (2015: 168). En otras palabras, la oferta de servicios gratuitos, a partir de algoritmos, permite la producción de un enorme volumen de datos con valor comercial. Google se convirtió, "a través de la recuperación de las huellas de sus usuarios, en una agencia de información y en cartógrafo de las identidades a nivel planetario" (Mattelart y Vitalis, 2015: 170). Han (2014: 102) es aún más enfático al comparar la actividad de Acxiom, Google y Facebook con la del servicio secreto del Estado: "los algoritmos de Facebook, de la bolsa y del servicio secreto llevan a cabo operaciones semejantes" y con frecuencia utilizan el mismo personal. Por lo tanto, la noción de economía de escala probablemente nunca haya sido tan verdadera, pues cada búsqueda aumenta la precisión de las respuestas propuestas por su algoritmo, dotando a las grandes firmas de un poder técnico y político incomparable. Cualificando la materialidad sobre la que se desarrollan las acciones estaríamos más próximos de entender tal poder.

El avance del dinero electrónico es otra manifestación técnica y política contemporánea que completa buena parte de los procesos de búsqueda a los que nos referimos. Al tiempo que las tarjetas de crédito y débito amplían sus estrategias de negocio, numerosos sistemas como Pay Pal también se expanden. Estas formas de transacción significan también el desarrollo de nuevas formas técnicas de control, fundadas en la georreferenciación y en la biometrización.

Con Lévy (1992: 230) diríamos que "la actividad técnica es una manera de entrelazar los humanos, los demás seres vivientes, las cosas, los sistemas de sistemas", y agrega "la técnica nos continúa", fundamentalmente a través de tecnologías intelectuales. Ya Prades (1992: 177) se había referido a esa omnipresencia del mundo de la técnica con el neologismo "tecnocosmos" porque, en palabras de Moles (1974: 64), "es lo artificial que es verdaderamente natural para el ser humano actual".

Corolario de la permanente renovación de las técnicas, la producción continua de normas conduce a lo que un autor como Dubet (2013: 324-325) identifica como un "universo de normas de producción y certificación de las mercancías" que impone a las actividades económicas numerosas "reglas jurídicas y técnicas". A ese "derecho 
compuesto" (Dubet, 2013: 325), porque es más un derecho de ingenieros, economistas y científicos que un derecho de juristas, el autor lo asocia con las máquinas. Y éstas, como bien señala Lévy (1992: 237) para referirse a los objetos técnicos, nos "hablan sobre todo en imperativo". El complejo STEM (science, technology, engineering, mathematics), al que se visualiza como creador de empleos en la actualidad y en un futuro próximo, es revelador de la relación intrínseca entre objetos técnicos y acción en el periodo actual. Sin embargo, algunas empresas comienzan a valorizar las denominadas capacidades blandas, es decir, las habilidades de comunicación y colaboración de sus empleados, que tampoco se hacen sin mediación tecnológica. Frente a muchos interrogantes sobre el trabajo en el mundo actual, el BID ha publicado el documento Robot-lution para discutir el impacto de la robotización en el trabajo, ya que no se prevé únicamente el desempleo de mano de obra de baja calificación, sino también el desempleo entre los white-collars, como ya había alertado Sennet (2009) al referirse a la medicina y las finanzas.

En definitiva, la técnica contemporánea se caracteriza por una expansión sistémica y relativamente autónoma fundada en el cálculo y, por lo tanto, permite hablar de una racionalización del espacio geográfico. La división territorial del trabajo hegemónica demanda técnicas de alto desempeño y velocidad, susceptibles de producir fluidez y autonomía en la esfera financiera o información económica ventajosa para realizar negocios de gran escala. En la producción moderna la demanda se orienta a técnicas rígidas e invasoras, que desprecian las solidaridades con técnicas más antiguas, mientras que, en la circulación, las grandes redes se vuelven indispensables. En todos esos casos la técnica es vista como un absoluto, como un dato inexorable, despertando formas de imitación en la dominación y contribuyendo para la producción de nuevos totalitarismos. Parafraseando a Gaudin (1978: 159) diríamos que son técnicas que responden a la "demanda del príncipe”, pues utilizan medios importantes y experts, amplían sin inventar, sirven al poder y les es difícil producir los objetos simples que liberan al hombre de sus esfuerzos cotidianos.

Pero existen, ciertamente, otras demandas técnicas que responden a las demás divisiones territoriales del trabajo. Se trata, en el decir de Gaudin (1978) y de Santos (2000), de técnicas divisibles, flexibles, dulces y dóciles, porque requieren inteligencia para funcionar y responden a usos y escalas distintos. Esa es, tal vez, su principal diferencia con las técnicas del periodo industrial. Las palabras de Berger (1964) renuevan su significado cuando asevera que los objetos consumen inteligencia durante toda su vida útil. A diferencia de los objetos dichos inteligentes en función de su grado de automatización, estas técnicas requieren inteligencia en sus combinaciones y aplicaciones. Podríamos aseverar con Martín-Barbero (2003) que son los usos populares de lo banal. Para diferenciarlas de las técnicas que responden a las demandas del príncipe, Gaudin (1978) las denomina técnicas populares porque, en su entender, resultan del hacer y de la imaginación de un gran número de personas, inventando objetos útiles para la vida cotidiana, aunque su éxito sea ocultado o confiscado. No son pocas las dimensiones de la vida social que revelan la necesidad de una mayor tecnificación, ya que podrían reducir o hacer desaparecer numerosas tareas que requieren considerable esfuerzo físico o conducen a una cierta alienación. Aún hoy, en un periodo de automatismos y de tecnologías espaciales, subsiste el transporte y movimiento de cargas a tracción humana o las tareas excesivamente repetitivas en ciertas máquinas. Entretanto, la liberación de estas tareas y la disposición de medios técnicos para un trabajo más creativo no serían posibles sin el desarrollo de mecanismos que eviten el desempleo y generen las condiciones políticas para los nuevos papeles sociales. 
Para algunos pensadores, estaríamos iniciando una era de lo artesanal, en la cual una técnica menos normatizada y más creativa puede apuntar soluciones a los problemas de la contemporaneidad. Es tal vez el caso del uso de impresoras 3D que han permitido fabricar manos ortopédicas, juegos de ajedrez para no videntes y placas de calles en sistema braille, aplicaciones que permiten conocer el territorio y vincular personas y actividades en nuevas interdependencias sociales y económicas. Por la utilización de objetos propios del sistema técnico de la globalización, estas manifestaciones se vuelven más visibles. Pero, además, existen innúmeros actores poco visibles que, en la co-presencia (Giddens, 1987), utilizan técnicas pretéritas o le dan otros usos a las técnicas contemporáneas para enfrentar la escasez. Particularmente en las periferias metropolitanas, servicios de reparación de vehículos y motos, electrodomésticos, computadoras y celulares, llenado de cartuchos de impresoras o fabricación artesanal de alimentos, son algunas de las infinitas manifestaciones creativas de supervivencia en la pobreza. En aquello que Sartre (1979) denomina condición de vecindad, el fenómeno técnico es vivido parcialmente pero es percibido como un mundo nuevo, constituyendo la base de la vida en común. En el lugar, existe una profunda interdependencia entre actores, pues unos trabajan para otros, aunque las relaciones sean desiguales. En esa realidad de razones plurales, la técnica, con edades y funcionalidades distintas, es constitutiva de la vida social y de sus temporalidades.

Por ello, parece importante ver la técnica no como absoluto, sino como un fenómeno. Más allá de la técnica dominante, cuya eficacia parece imposible de ser impugnada a pesar de las crisis, existe un abanico de formas de hacer y de medios técnicos que puede ser reconocido a partir de un pensamiento crítico. Se trataría de aprehender las cosas en los lugares más la vida que les da sentido, en un proceso de transformación recíproca entre objetos y acciones, entre condición y actualidad. 


\section{Q Bibliografía}

»Akrich, M. (1987). Comment decrire les objets techniques? Techniques et Culture, 9, 49-63.

» Arendt, H. (2005). De la historia a la acción. Buenos Aires: Paidós.

»Auzias, J.-M. (1971). La philosophie et les techniques. Paris: PUF, 2 Ed.

》Bagú, S. (1986). Tiempo, realidad social y conocimiento. México: Siglo XXI, 11 Ed.

" Barraclough, G. (1983). Introdução à Histórica Contemporânea. Rio de Janeiro: Zahar, $5 \mathrm{Ed}$.

» Berger, G. (1964). Phénoménologie du Temps et Prospective. Paris: PUF.

»Braidotti, R. (2015). Lo Posthumano. Barcelona: Gedisa.

» Braudel, F. (1973). Las civilizaciones actuales. Madrid: Tecnos.

» Coriat, B. (1976). Science, Tecnique et Capital. Paris: Seuil.

»Debray, R. y Finkielkraut, A. (1994). Les techniques et l'humanisme. En F. Bayle et al. (Eds.), L'empire des techniques (pp. 231-251). Paris: Seuil. (Entretien: Ruth Scheps).

»Dubet, F. (2013). El trabajo de las sociedades. Buenos Aires: Amorrortu.

》Elias, N. (2010). Sobre el tiempo. México: FCE, 3 Ed. (Publicación original 1984).

》Ellul, J. (1968). A técnica e o desafio do século. Rio de Janeiro: Paz e Terra.

» Fel, A. (1978). La géographie et les techniques. En Histoire des Techniques (pp. 1062-1110). Paris: Encyclopédie de la Pléiade.

„ Friedmann, G. (1966). Sept Études sur l'hommeet la technique. Paris: Denoel/ Gonthier.

» Gaudin, T. (1978). L'écoute des silences, les institutions contre l'innovation? Paris: Union Générale des Éditions.

" George, P. (1974). L'ère des techniques, constructions ou destructions? Paris: PUF.

》Giddens, A. (1987). La constitution de la société. Éléments de la théorie de lastructuration. Paris: PUF.

» Gille, B. (1978). Histoire des techniques. Paris: Encyclopédie de La Pléiade.

" Gras, A. (1993). Grandeur et dépendance. Sociologie des macro-systèmes techniques. Paris: PUF.

" Gurvitch, G. (1962). Dialectique et Sociologie. Paris: Flammarion.

»Han, B.-Ch. (2014). En el enjambre. Barcelona: Herder.

»Heidegger, M. (1958). Essais et Conferences. Paris: Gallimard.

» Heidegger, M. (1975). La pregunta por la cosa. La doctrina kantiana de los principios trascendentales. Barcelona: Orbis.

» Hughes, T. P. (2008). La evolución de los grandes sistemas tecnológicos. En H. 
Thomas y A. Buch (Eds.), Actos, actores y artefactos. Sociología de la tecnología (pp. 101-145). Bernal: Universidad Nacional de Quilmes.

»IDB (2017). Robotlution. The future of work in Latina American Integration 4.0. I\&T, $42,21$.

" Joerges, B. (1988). Large Technical Systems: concepts and issues. En R. Maynz y T. P. Hughes (Eds.), The Development of Large Technical Systems. Frankfurt: Westview Press, Boulder Co., Campus Verlag.

» Ladrière, J. (1977). Les enjeux de la rationalité. Le défi de la science et de la technologie aux cultures. Paris: Aubier-Montaigne/Unesco.

» Latour, B. (2008). Reensamblar lo social. Una introducción a la teoría del actor-red. Buenos Aires: Manantial.

» Lévy, P. (1992). Quelle critique de la technique? En J. Prades (Ed.), La Technoscience. Les fractures des discours (pp. 229-245). Paris: L'Harmattan.

» Martín-Barbero, J. (2003). Ofício de cartógrafo. Travesías latinoamericanas de la comunicación en la cultura. Bogotá, Fondo de Cultura Económica.

» Mattelart, A. y Vitalis, A. (2015). De Orwell al cibercontrol. Barcelona: Gedisa.

» Moles, A. (1974). Phénoménologie de l' action. Les sciences de l' action. Paris: CEPL.

» Ortega y Gasset, J. (1957). Meditación de la Técnica. Vicisitudes de las ciencias. Bronca en la Física. Madrid: Revista de Occidente, 3 Ed.

"Perrin, J. (1992). L'importance décisive des modèles d'organisation. En J. Prades (Ed.), La Technoscience. Les fractures des discours (pp. 99-119). Paris: L'Harmattan.

"Picon, A. (1994). Le dynamisme des techniques. En F. Bayle et al. (Ed.), L'empire des techniques (pp. 25-37). Paris: Seuil. (Entretien: Ruth Scheps).

» Prades, J. (1992). La Technoscience. Les fractures des discours. Paris: L'Harmattan.

》 Reclus, É. (1985). A complexidade da produção do espaço geográfico. En M.C. Andrade, Élisée Reclus (pp. 56-60). São Paulo: Ática.

» Ribeiro, D. (1975). 0 processo civilizatório. Rio de Janeiro: Civilização Brasileira, 3 Ed.

》Santos, M. (1985). Espaço e Método. Nobel, São Paulo.

»Santos, M. (1994). $O$ retorno do território. En M. Santos, M.A.A. Souza y M.L. Silveira (Eds.), Território: Globalização e Fragmentação (pp. 15-20). São Paulo, Hucitec.

»Santos, M. (2000). La naturaleza del espacio. Técnica y tiempo. Razón y emoción. Barcelona: Ariel. (Publicación original en portugués 1996).

»Santos, M. y Silveira, M. L. (2001). O Brasil: território e sociedade no início do século XXI. Rio de Janeiro: Record.

»Sartre, J.-P. (1968). El ser y la nada. Ensayo de ontología fenomenológica. Buenos Aires: Losada, 2 Ed.

»Sartre, J.-P. (1979). Crítica de la Razón Dialéctica. Precedida de Cuestiones de Método. Buenos Aires: Losada, 3 Ed. 
»Sennett, R. (2009). $O$ artífice. Rio de Janeiro: Record.

"Sigaut, F. (1994). La technologie, une science humaine. En F. Bayle et al. (Ed.). L'empire des techniques (pp. 51-61). Paris: Seuil. (Entretien: Ruth Scheps).

» Silveira, M. L. (2012). El fenómeno técnico en la comprensión de la historia del territorio. Espaciotiempo Revista Latinoamericana de Ciencias Sociales y Humanidades, 5(7), 51-64.

"Silveira, M. L. (2014). El territorio usado, un caleidoscopio de divisiones del trabajo. Revista Geográfica del Sur, 5(7), 15-34.

»Simmel, G. (2002). Sobre la individualidad y las formas sociales. Bernal: Universidad Nacional de Quilmes.

»Simmel, G. (2006). Problemas fundamentales de la Filosofía. Andalucía: Espuela del Plata.

»Simondon, G. (1989). Du mode d'existence des objets techniques. Paris: Aubier.

»Smith, N. (1990). Uneven Development. Oxford: Basil Blackwell, 2 Ed.

» Tarde, G. (2011). Creencias, deseos, sociedades. Buenos Aires: Cactus.

»Torres Ribeiro, A. C. (2012). Por uma sociologia do presente. Ação, técnica, espaço. Rio de Janeiro: Letra Capital.

»Van Dijck, J. (2016). La cultura de la conectividad: una historia crítica de las redes sociales. Buenos Aires: Siglo XXI.

María Laura Silveira / maria.laura.silveira.1@gmail.com

Profesora y Licenciada en Geografía (Universidad Nacional del Comahue) y Doctora en Geografía Humana (Universidade de São Paulo). Fue Profesora en la Universidad de São Paulo e Investigadora del CNPq. Actualmente es Investigadora Principal del CONICET con sede en el Instituto de Geografía, de la Facultad de Filosofía y Letras, Universidad de Buenos Aires. Ha desarrollado investigaciones y ha publicado en las áreas de Geografía Urbana, Estudios Territoriales y Epistemología de la Geografía. 